\title{
Profile of ranibizumab: efficacy and safety for the treatment of wet age-related macular degeneration
}

This article was published in the following Dove Press journal:

Therapeutics and Clinical Risk Management

10 July 2012

Number of times this article has been viewed

\section{Youxin Chen}

Fei Han

Department of Ophthalmology, Peking Union Medical College Hospital, Peking Union Medical College and Chinese Academy of Medical Sciences, Beijing, China
Correspondence: Youxin Chen Department of Ophthalmology, Peking Union Medical College Hospital, No I Shuai Fu Yuan, Wang Fu Jing St, Beijing 100730, China Tel +861069156358

Fax +86I069I56565

Email chenyouxin@medmail.com.cn
Abstract: Wet age-related macular degeneration (AMD) causes severe vision loss due to the development of choroidal neovascularization (CNV). The critical role of vascular endothelial growth factor in the pathogenesis of CNV is well understood. Ranibizumab plays an inhibitory role with CNV and reduces vascular permeability by binding to vascular endothelial growth factor. Intravitreal ranibizumab reduces the risk of visual acuity (VA) loss and increases the chance of VA gain compared with no treatment or photodynamic therapy for CNV in AMD. Some high-quality research has shown that the optimal timing for ranibizumab treating wet AMD is the first 3 months. It is recommended that ranibizumab is intravitreally injected monthly in the initiation for at least 3 months. Subsequent managing of regimens should be made dependent on the VA change, fundus examination, and image of optical coherence topography. An individualized strategy or combined method with photodynamic therapy is beneficial to the active lesion in the consecutive treatment of ranibizumab for $\mathrm{CNV}$, and may be a good choice in order to decrease injection times. Regarding the safety profile, ranibizumab has been well tolerated in clinical trials. The principal ocular adverse event detected in clinical trials is a low frequency of ocular inflammation. Key serious ocular adverse events occurred in $<5 \%$ of ranibizumabtreated patients in large-scale clinical trials. It appears unlikely that treatment with ranibizumab increases the risk of vascular events significantly. Less frequent injections on an as-needed schedule, based on monthly monitoring may have the most optimal risk:benefit ratio.

Keywords: age-related macular degeneration, choroidal neovascularization, ranibizumab, efficacy, safety

\section{Introduction}

Age-related macular degeneration (AMD) is considered to be the leading cause of blindness and visual handicap in the elderly population in the developed world., ${ }^{1,2}$ Wet or neovascular AMD accounts for approximately $10 \%$ of all macular degeneration cases but $90 \%$ of blindness from AMD. ${ }^{3}$ The word "neovascular" describes the abnormal growth of new blood vessels from the choroid into or underneath the retina. The main pathogenesis of vision loss in wet AMD is the development of choroidal neovascularization (CNV). Vascular endothelial growth factor-A (VEGF-A) is the key cytokine closely associated with the growth and permeability of neovascular vessels. The central role of VEGF in the pathogenesis of CNV has been well established. ${ }^{4-6}$ Ranibizumab (Lucentis ${ }^{\circledR}$; Genentech Inc, South San Francisco, CA) is the Fab fragment of a recombinant, humanized, monoclonal antibody that binds to all forms of VEGF-A, thereby inhibiting angiogenesis and reducing vascular permeability. ${ }^{7}$ Ranibizumab (0.5 mg monthly, intravitreal injection [IVI]) was approved for use in 
neovascular AMD in the US in 2006, in Europe in 2007, and in Japan in 2009. This profile discusses and summarizes the efficacy and safety profile of ranibizumab, based on currently available data from clinical trials involving patients with neovascular AMD.

\section{Pharmacodynamic and pharmacokinetic profile of ranibizumab}

\section{Pharmacodynamic profile}

Ranibizumab is a recombinant, humanized immunoglobulin $\mathrm{G}_{1}$ kappa murine monoclonal antibody fragment. ${ }^{8-11}$ It is produced in an Escherichia coli expression system and has a molecular weight of $\sim 48 \mathrm{kDa} .{ }^{8}$ By binding to the receptorbinding site of active forms of VEGF-A (eg, VEGF ${ }_{110}$, $\mathrm{VEGF}_{121}$, and $\mathrm{VEGF}_{165}$ ), ranibizumab prevents VEGF-A from interacting with its receptors located on the endothelial cell surface, thereby reducing endothelial cell proliferation, vascular leakage, and angiogenesis. In vitro, ranibizumab binds with high affinity to the active forms of VEGF-A (apparent dissociation rate constant $\leq 10^{-5}$ ) and inhibits human umbilical vein endothelial cell proliferation induced by these active isoforms of VEGF-A in a concentration-dependent manner. ${ }^{12}$ In vivo, ranibizumab inhibits the increase in vascular permeability induced by VEGF-A in a dose-dependent fashion (mean $\left.\mathrm{IC}_{50} 0.4-1.2 \mathrm{nmol} / \mathrm{L}\right){ }^{12}$

\section{Pharmacokinetic profile}

Given the intravitreal route of administration, systemic exposure to ranibizumab is expected to be negligible. ${ }^{13}$ The maximum serum concentration of ranibizumab after $0.5 \mathrm{mg} / \mathrm{month} /$ eye, which was attained approximately 1 day after administration, was $0.79-2.9 \mathrm{ng} / \mathrm{mL}$. Based on a population pharmacokinetic model generated by the manufacturer, vitreal ranibizumab concentrations are predicted to be 90,000- and 140,000-fold higher than plasma ranibizumab concentrations after $0.3 \mathrm{mg}$ and $0.5 \mathrm{mg}$ doses, respectively. ${ }^{13-15}$ That is clinically important because extraocular VEGF-A is necessary for normal physiologic functions. Over the ranibizumab dose range of $0.05-1.0 \mathrm{mg} /$ eye, the maximum observed serum concentration was dose-proportional. In recipients of ranibizumab $0.5 \mathrm{mg}$, the mean estimated vitreous elimination half-life of ranibizumab was $\sim 9$ days, based on a neovascular AMD population pharmacokinetic analysis and the disappearance of the drug from the plasma after a $0.5 \mathrm{mg}$ dose. ${ }^{9}$ Creatinine clearance was found to be the most significant covariate for ranibizumab clearance; however, the decrease in ranibizumab clearance in patients with mild-to-moderate renal impairment was not associated with an increase in systemic exposure that would be clinically relevant, and dosage adjustment is not needed in this population. ${ }^{9}$

\section{Therapeutic efficacy}

The results of randomized controlled clinical trials of ranibizumab for the treatment of neovascular AMD established a new standard of care with the prospect of improved vision in many patients. Subsequent trials have explored different strategies to increase response rates and reduce treatment frequency.

\section{Ranibizumab monotherapy for neovascular AMD}

Several studies indicate that untreated subfoveal CNV may grow quickly, on average around $10 \mu \mathrm{m}$ per day. ${ }^{16}$ Successful treatment of neovascular AMD requires small intervals between diagnosis and first ranibizumab injection. Treatment as early as possible, and at a maximum of within 2 weeks of diagnosis, is ideal. Durations longer than 1 month risk increasing visual loss. ${ }^{17-19}$

Ranibizumab initiation with three consecutive monthly injections appears optimal, as this is when the majority of patients experienced most VA gain in all studies. MARINA (Minimally Classic/Occult Trial of the Anti-VEGF Antibody Ranibizumab in the Treatment of Neovascular Age-related Macular Degeneration) and ANCHOR (Anti-VEGF Antibody for the Treatment of Predominantly Classic Choroidal Neovascularization in Age-related Macular Degeneration) were the randomized, double-blinded, controlled, multicenter Phase III trials with monthly injections throughout the whole treatment period. MARINA compared ranibizumab $0.3 \mathrm{mg} / \mathrm{month}(\mathrm{n}=238)$ or $0.5 \mathrm{mg} / \mathrm{month}(\mathrm{n}=240)$ with sham injection $(n=238)$ for 2 years in patients with minimally classic or occult subfoveal CNV secondary to AMD. ${ }^{20}$ ANCHOR compared ranibizumab $0.3 \mathrm{mg} / \mathrm{month}(\mathrm{n}=140)$ or $0.5 \mathrm{mg} / \mathrm{month}(\mathrm{n}=140)$ with veteporfin photodynamic therapy (PDT) given at baseline and every 3 months as required $(n=143)$ for 2 years in patients with predominantly classic CNV lesions. ${ }^{21,22}$ Most VA improvement was seen during the initial 3-month phase, with subsequent injections appearing to maintain the achieved benefit. Moreover, the VA improvements observed with ranibizumab in the first 3 months were sustained (and some additional improvement was seen) over the full 24-month trial period. Monthly ranibizumab $0.3 \mathrm{mg}$ or $0.5 \mathrm{mg}$ was associated with mean gains of 6.5-11.3 letters at 1 year and 5.4-10.7 letters at 2 years, versus mean losses of 10.4 and 14.9 letters with 
sham injection at 1 and 2 years and a mean loss of 9.5 and 9.8 letters with PDT at 1 and 2 years. In addition, ranibizumab treatment was associated with arrested growth of, and leakage from, CNV; the improvement in foveal centerpoint thickness was also observed. According to these two large-scale, randomized clinical trials, the standard regimen of ranibizumab for wet AMD is monthly treatments administered for 2 years. However, the frequency of therapy raised secondary issues, such as the logistical feasibility of the regimen, patients' compliance, and the costs of the monthly treatment, became increasingly apparent. Adopting a variable ranibizumab dosing strategy guided by VA assessment and optical coherence tomography (OCT) are now being tried to reduce the frequency of injections.

The first alternative strategy to monthly injections was tested in the PIER (A Phase IIIb, Multicenter, Randomized, Double Masked, Sham Injection Controlled Study of the Efficacy and Safety of Ranibizumab in Subjects with Subfoveal Choroidal Neovascularization with or without Classic Choroidal Neovascularization Secondary to Age-related Macular Degeneration) study and consisted of an initiation phase followed by a fixed dose of intravitreal ranibizumab every 3 months. ${ }^{23,24}$ Ranibizumab demonstrated a clinically meaningful benefit in mean VA change from baseline compared with sham at 12 and 24 months. However, unlike the results from MARINA and ANCHOR, there was no overall vision improvement over the 24 -month trial period. It must be emphasized that there was an initial improvement in the initiation phase, which was later lost in the period of quarterly maintenance injections. However, subgroup analysis indicated that an alternative regimen could benefit some patients: $40 \%$ of patients in the PIER study had an initial improvement in VA following the first 3-monthly injections, which was maintained by quarterly fixed-dose injections, resulting in a gain of about nine letters in 12 months. In the SAILOR (Safety Assessment of Intravitreous Lucentis for AMD) study, ranibizumab doses of 0.3 and $0.5 \mathrm{mg}$ were evaluated without a control group. ${ }^{25}$ The treatment schedule consisted of an initiation phase followed by quarterly monitoring visits and injections, the on-demand or pro renata $(\mathrm{PRN})$ retreatment was based on VA (more than five-letter loss from the previous highest VA score) and OCT criteria (>100 $\mu \mathrm{m}$ increase in CRT from the previous lowest measurement). The overall results of this study were similar to those in the PIER study. There was no improvement versus baseline. This suggested that quarterly visits were insufficient to monitor and capture disease progression, and more frequent monitoring is needed.
These results encouraged the search for further alternative strategies. In the EXCITE (Efficacy and Safety of Ranibizumab in Patients with Subfoveal Choroidal Neovascularization Secondary to Age-related Macular Degeneration) study, patients were given an injection each month for the first 3 months. One group of patients was retreated monthly (ranibizumab $0.3 \mathrm{mg}$ ), whereas the other two groups were given 0.3 or $0.5 \mathrm{mg}$ ranibizumab quarterly over 12 months. ${ }^{26}$ The results confirmed that the monthly dosing regimen was more effective than the quarterly one. At month 12 compared with month 3 , the VA gain was slightly decreased with quarterly dosing (by 22.2 and 23.1 letters with $0.3 \mathrm{mg}$ and $0.5 \mathrm{mg}$ of ranibizumab, respectively) but was slightly increased (by +0.9 letters) with $0.3 \mathrm{mg}$ of ranibizumab monthly. Similar to findings in the PIER study, $43 \%$ of quarterly regimen patients in the EXCITE study obtained a gain in VA similar to that of the monthly regimen patients.

The PrONTO (Prospective OCT Imaging of Patients with Neovascular AMD Treated with Intraocular Lucentis) study examined a PRN regimen with monthly visits in 40 patients. $^{27}$ Following the initiation phase, monitoring was given monthly, and the retreatment was based on vision, fundus examination, and OCT criteria. A total of $82 \%$ of patients experienced no loss of vision, and there was an average VA gain of 9.3 letters after the first 3 months, which remained stable for the full 12 months. These results were similar to those obtained in MARINA and ANCHOR, but instead of 13 injections an average of only 5.9 injections was given during the PrONTO study over a period of 1 year, which suggests that flexible OCT-guided retreatment could sustain visual gain with fewer injections. However, this study did have major limitations: it was a small, open-label, centralized study that lacked a control group. A larger-scale trial called SUSTAIN (Study of Ranibizumab in Patients with Subfoveal Choroidal Neovascularization Secondary to Age-related Macular Degeneration) investigated an individualized ranibizumab PRN regimen in 513 patients. ${ }^{28}$ The regimen strategies were similar to those in the PrONTO study. The VA results were positive: with an average of 5.6 injections, the mean change in patient VA at the end of the initiation phase was +5.8 letters, and after 12 months the improvement was +3.6 letters from baseline, which was slightly reduced.

The CATT (Comparison of Age-related Macular Degeneration Treatment Trials) study is a multicenter randomized clinical trial to access the efficacy of ranibizumab and bevacizumab when administered monthly or as needed for 2 years and to describe the impact of switching to as-needed treatment after 1 year of monthly treatment. ${ }^{29}$ At enrollment, patients 
were assigned to four treatment groups defined by drug (ranibizumab or bevacizumab) and dosing regimen (monthly or as needed). At 1 year, patients initially assigned to monthly treatment were reassigned randomly to monthly or as-needed treatment. Patients receiving the as-needed dosing regimen were evaluated for treatment every 4 weeks and were treated when fluid was present on OCT or when there was new or persistent hemorrhage or decreased VA relative to previous visits. We concentrate only on ranibizumab here. At 2 years, as-needed dosing of ranibizumab produced 2.4 letters less mean gain than monthly dosing $(P=0.046)$. This may be the result of more lesion growth, fluorescein leakage, and residual fluid on OCT in eyes in the as-needed group. Switching from monthly to as-needed treatment resulted in greater mean decrease in vision during year 2. CATT is the most recent landmark trial for wet AMD, which demonstrates that treatment as needed resulted in less gain in VA whether instituted at enrollment or after 1 year of monthly treatment.

Summarizing the aforementioned clinical trials, apart from the limited PrONTO study, alternative ranibizumab regimens have not achieved the same levels of efficacy as the monthly injections studied in MARINA and ANCHOR. The goal of maintaining VA after the initiation phase by pre-empting active disease without monthly injections has yet to be achieved. Monés ${ }^{30}$ proposed a new treatment regimen with the aim of rationalizing treatment frequency without compromising improvements in vision, the FUSION regimen. This consists of an initiation phase followed by PRN retreatment combined with fixed injections after a period of disease inactivity of 2-4 months (depending on the time elapsed since the last injection), but a randomized clinical trial for comparing monthly, PRN, and FUSION regimens is necessary.

According to the evidence from the clinical trials, we suggest the following recommendations for ranibizumab therapy. Commencement of treatment (level I evidence):

- Treatment should be commenced as soon as possible after diagnosis. The recommended time interval permitted before treatment initiation in the clinical studies was $\leq 14$ or 28 days. ${ }^{31}$

- $0.5 \mathrm{mg}$ of ranibizumab should be initiated with at least three consecutive monthly IVIs. ${ }^{32}$

- Before administering ranibizumab at months 1 and 2, follow-up examination is recommended: history, VA assessment, and slit-lamp fundus examination and OCT. Fluorescein angiography is recommended for the patients with significant or unexplained vision loss.
Maintenance of treatment:

- Level I evidence indicated that a monthly regimen of ranibizumab IVI had the best VA outcomes in the clinical trials.

- When a monthly regimen is not possible, a flexible strategy with monthly monitoring is feasible, but benefits could be less than with the monthly treatment.

- Monthly monitoring is recommended, particularly during the first 12 months, in order to detect active disease. If active disease is present or recurs, additional treatment should be initiated quickly; otherwise, retreatment is not necessary. If the clinical signs remain stable for a longer period, extending the follow-up intervals may then be justified.

\section{Ranibizumab in combination with PDT}

Ranibizumab in combination with PDT is another way to optimize treatment. It has been studied in the FOCUS (RhuFab V2 Ocular Treatment Combining the Use of Visudyne to Evaluate Safety) and PROTECT (Same-day Administration of Verteporfin and Ranibizumab $0.5 \mathrm{mg}$ in Patients with Choroidal Neovascularization due to Age-related Macular Degeneration) studies. The results of the FOCUS trial indicated that ranibizumab + PDT was more effective than PDT alone. ${ }^{33}$ At month $24,88 \%$ of ranibizumab/PDT patients had lost $<15$ letters from baseline VA (vs $75 \%$ for PDT alone), $25 \%$ had gained $\geq 15$ letters (vs $7 \%$ for PDT alone), and the two treatment arms differed by 12.4 letters in mean VA change. Moreover, the combination group exhibited less lesion growth and greater reduction of CNV leakage and subretinal fluid accumulation, and required fewer PDT retreatments, than the PDT alone group. The PROTECT study evaluated the combination of PDT administered at baseline and as needed at months 3, 6, and 9, and ranibizumab $0.5 \mathrm{mg}$ administered at baseline and months 1, 2, and $3 .{ }^{34}$ Baseline and month 3 ranibizumab treatments were administered on the same day as, and at least 1 hour after, PDT. It showed that PDT/ranibizumab was associated with CNV occlusion, reduced edema, improved visual function, and retinal sensitivity. The 12-month primary analysis of the multicenter randomized MONT BLANC study confirmed the noninferiority of combination therapy over ranibizumab monotherapy ${ }^{35}$ Ranibizumab in combination with PDT may result in stable VA with substantially fewer IVIs.

In summary, combination PDT/ranibizumab therapy has been used in an effort to reduce the number of IVIs in patients who may have difficulty with follow-up, or in those who reject repeated IVIs. It has been determined that the 
number of injections required was only slightly reduced and not statistically significant. Nevertheless, given the practical considerations of individual patients, this treatment can be beneficial in selected cases.

\section{Safety and tolerability}

Safety issues with ranibizumab IVI include local ocular adverse events from the drug or the injection, as well as potential systemic adverse events of the drug. When considering the safety profile of ranibizumab, it is important to note that clinical trials may underestimate the incidence of adverse events, especially if the hazard rate for an event increases over time.

\section{Ocular safety}

It was concluded that the main ocular safety risks of ranibizumab therapy were due to the IVI itself. The most common adverse ocular reactions reported in patients receiving ranibizumab during clinical trials included intraocular inflammation, increased intraocular pressure, conjunctival hemorrhage, and cataract. Serious adverse events considered to be potentially related to intravitreal ranibizumab treatment included endophthalmitis, uveitis, vitreous hemorrhage, rhegmatogenous retinal detachment, retinal tear, and lens damage.

Tolerability data for ranibizumab injection at monthly intervals were obtained from the phase III trials (MARINA and ANCHOR). ${ }^{20-22}$ Serious ocular adverse events were infrequent in both studies: $3.8 \%$ ( $0.3 \mathrm{mg}$ of ranibizumab), $5.0 \%$ ( $0.5 \mathrm{mg}$ of ranibizumab), and $1.3 \%$ (sham) in MARINA, and $2.9 \%$ (0.3 $\mathrm{mg}$ of ranibizumab), 3.6\% (0.5 $\mathrm{mg}$ of ranibizumab), and 0.7\% (PDT) in ANCHOR. Similar incidences of presumed endophthalmitis have been reported in the two studies ( $1 \%$ in MARINA, $1.1 \%$ in ANCHOR); rates per injection were both $0.05 \%$. Rates of intraocular inflammation were $11.7 \%-20.8 \%$ in ranibizumab-treated groups, $12.7 \%$ in the sham group, and $3.5 \%$ in the PDT group, with most of the inflammation designated as trace or $1+$. Within 60 minutes of injection, intraocular pressure increased by $\geq 30 \mathrm{mmHg}$ in $9 \%-18 \%$ of ranibizumab $0.3 \mathrm{mg}$ or $0.5 \mathrm{mg}$ recipients, $4 \%$ of PDT recipients, and $3 \%$ of sham injection recipients. The mean increases from preinjection to postinjection were small and transient in all groups and unlikely to require prolonged monitoring. There was no increase in the incidence of cataract in ranibizumab recipients (15.5\% in both groups) compared with those receiving sham injections (15.7\%) in MARINA; by contrast, in the ANCHOR trial, cataract formation was more frequent with the ranibizumab group $(16.8 \%$ in the
$0.3 \mathrm{mg}$ group and $20 \%$ in the $0.5 \mathrm{mg}$ group) than in the PDT group $(10.5 \%)$. The difference was statistically significant for $0.5 \mathrm{mg}$, indicating the possibility that ranibizumab may accelerate the progression of cataract. Further investigation of the incidence of cataract is warranted.

The ocular safety of ranibizumab administered at a reduced frequency (after a 3-month initial phase of monthly treatment) has been evaluated in the PIER, SAILOR, SUSTAIN, and EXCITE studies. In the PIER study, patients received fixed quarterly dosing of ranibizumab in the maintenance phase. ${ }^{23,24}$ This regimen was generally well tolerated. Serious ocular hemorrhage occurred in two patients in each of the sham (3.2\%) and ranibizumab $0.3 \mathrm{mg}$ (3.4\%) groups. Serious macular edema occurred in two patients $(3.2 \%)$ in the sham group and one patient $(1.7 \%)$ receiving ranibizumab $0.3 \mathrm{mg}$. No other serious ocular adverse events occurred. The majority of patients ( $95 \%)$ in all treatment groups had no evidence of intraocular inflammation. The incidences of cataract formation were similar in the three groups $(5.1 \% \sim 6.5 \%)$. Twelve-month results of the SUSTAIN, EXCITE, and SAILOR studies show similar incidences of ocular adverse events to other clinical studies. ${ }^{25,26,28}$

A number of case series and retrospective studies have provided further insight into the ocular safety profile of ranibizumab. A retrospective analysis of 14,320 IVIs of ranibizumab revealed an incidence of endophthalmitis of $0.02 \%$, which is consistent with the rate of $0.05 \%$ per injection in MARINA and ANCHOR. ${ }^{36}$ The incidences of retinal pigment epithelial tear after intravitreal anti-VEGF therapy reported in the retrospective case reviews were $0.6 \% \sim 2.2 \%{ }^{37}$ Some researchers concluded that patients with high-risk lesions, especially large, irregular pigment epithelial detachment associated with $\mathrm{CNV}$, should be monitored for the development of retinal pigment epithelial tears. ${ }^{38,39}$ The incidence of retinal tears in clinical practice is possibly higher than that in MARINA and ANCHOR, given the exclusion of patients with large lesions from these studies.

\section{Systemic safety}

The many roles that VEGF plays in physiologic processes give reason for concern that its inactivation could have potentially serious systemic consequences. VEGF inhibitors that cross into the general circulation can compromise functions that rely on VEGF outside of the eye, such as wound healing and the formation of new blood vessels around the heart or brain in cases of ischemia. ${ }^{40}$ The overall incidence of systemic adverse events in the ranibizumab trials was low, but the apparent increase in nonocular hemorrhages and 
thromboembolic events suggested potential increased risks with ranibizumab treatment.

\section{Nonocular hemorrhage}

The 2-year incidences of nonocular hemorrhage in MARINA were $9 \%$ for the combined ranibizumab groups $(0.3 \mathrm{mg}$ and $0.5 \mathrm{mg}$ ), compared with $5.5 \%$ for sham-treated patients. ${ }^{20}$ In the ANCHOR trial, a higher incidence of nonocular hemorrhage was evident at 1 year, with a combined incidence in the groups receiving both ranibizumab and PDT of $5.8 \%$, compared with $2.1 \%$ in patients treated with only PDT. ${ }^{21}$ At 2 years the respective frequencies were $9.0 \%$ and $4.9 \% .{ }^{22}$ Gillies and Wong, ${ }^{41}$ using 2-year data for MARINA and 1-year data for ANCHOR, indicated that there was a significant increase in the occurrence of nonocular hemorrhage when data from the two trials were combined $(7.8 \%$ in the ranibizumab groups vs $4.2 \%$ in the controls; $P=0.01$ ). Reported nonocular hemorrhagic events included ecchymosis, gastrointestinal hemorrhages, hematoma, vaginal hemorrhages, and subdural hematomas. The rates of nonocular hemorrhage in the other clinical studies were consistent with those seen in MARINA and ANCHOR.

\section{Arterial thromboembolic events}

Arterial thromboembolic events (ATEs) were defined according to the Antiplatelet Trialists' Collaboration group, including nonfatal myocardial infarction, nonfatal ischemic stroke, nonfatal hemorrhagic stroke, or death owing to vascular or unknown causes. In MARINA and ANCHOR, there was no statistical difference in cumulative ATEs across treatment groups. The incidences were $4.4 \% \sim 5.0 \%$ (ranibizumab therapy), 3.8\% (sham), and 4.2\% (PDT). ${ }^{20-22}$ No ATEs occurred during year 1 of the PIER study. The 12-month results of the SUSTAIN study showed that most frequent nonocular serious adverse events were cardiac failure $(1.2 \%)$ cerebral accident, and myocardial infarction (1\%). ${ }^{28}$ The safety analysis of the 1 -year SAILOR trial revealed that there was an increased risk of stroke in the higher-dose group $(1.2 \%$ and $0.7 \%$ for the $0.5 \mathrm{mg}$ and $0.3 \mathrm{mg}$ ranibizumab groups, respectively), but the difference was not statistically significant. ${ }^{25}$ Patients with a prior history of stroke appeared to be at higher risk, but no increased risk of myocardial infarction or vascular death was identified. Reassessment of the SAILOR trial findings is needed using other studies and cohorts. In a combined analysis of SAILOR and the 1-year data from ANCHOR, MARINA, and PIER, ATEs occurred in 2.5\% of ranibizumab-treated patients. ${ }^{42}$ Similarly, another metaanalysis of combined data from all ranibizumab trials of patients receiving monthly injections $(0.3 \mathrm{mg}$ or $0.5 \mathrm{mg})$ and those receiving sham treatment showed that the incidence of cardiovascular accidents was $2.2 \%$ in the ranibizumab-treated subjects versus $0.7 \%$ in sham-treated subjects $(P=0.045) .{ }^{43}$ There was no apparent association between ranibizumab injections and myocardial infarction.

The incidence of ATEs in clinical studies should be considered in the context of the risk of such events in the general population of older individuals and specifically in patients with AMD. Patients with AMD already are at higher risk of cardiovascular disease than the general population, by virtue of their age and the association of AMD with systemic hypertension. ${ }^{44}$ In addition, ischemia is believed to be one of the key factors involved in the pathogenesis of CNV due to AMD. The Framingham-derived risk of cardiovascular events in individuals $>50$ years of age is higher than the observed incidence in the evidence level I clinical studies of ranibizumab. ${ }^{45}$ Possible reasons include self-selection bias in the enrollment of patients and the exclusion of patients with certain conditions.

\section{Immunoreactivity}

Low titers of antibodies to ranibizumab were detected in approximately $1 \%-8 \%$ of ranibizumab-treated patients in MARINA, ANCHOR, PIER, and FOCUS. ${ }^{20-24,33}$ Antibody presence may lead to a variety of effects, such as anaphylaxis, modifications in the pharmacokinetics of the drug, reduction in drug efficacy, and cross-reactivity with endogenous proteins. The clinical significance of immunoreactivity to ranibizumab is currently unclear. Although the numbers were small, subjects in the ANCHOR study who were immunoreactive at baseline or during treatment were more likely to develop ocular inflammation than those who were not. ${ }^{21,22}$ There was no notable relationship between immunoreactivity and nonocular adverse events or VA results. Further monitoring of possible clinical signs attributable to immunoreactivity should be performed.

\section{The long-term efficacy and safety profile of ranibizumab}

In the HORIZON (An Open-label Extension Trial of Ranibizumab for Choroidal Neovascularization Secondary to Agerelated Macular Degeneration) extension study, patients who had completed the 2-year MARINA, ANCHOR, or FOCUS trials could continue treatment with ranibizumab therapy $(0.5 \mathrm{mg})$ on an as-needed basis. ${ }^{46}$ With less frequent follow-up leading to less treatment, there was an incremental decline of the VA gains achieved with monthly treatment, 
presumably related to undertreatment in the extension period. A total of $69 \%$ and $70 \%$ of patients needed some additional treatments in the third and the fourth year, respectively; overall, better VA and anatomical outcomes after 2 years predicted a longer time to retreatment in this period. No new safety issues emerged, and repeated IVIs of ranibizumab were well tolerated for 4 or more years. These findings demonstrate the long-term safety of ranibizumab when continued at less frequent dosing in years 3 and 4 . The ongoing SECURE extension trial of SUSTAIN and EXCITE will also provide useful information about long-term efficacy and safety of ranibizumab.

\section{Patient satisfaction}

AMD has a deleterious impact on patients' quality of life. The increase in distance vision after ranibizumab treatment is well established in clinical trials. But near vision in general and reading in particular is of great importance and interest to elderly people; they often indicate reading difficulties as the first symptom of AMD. A recent study in Sweden showed significant improvement in important visual qualities, such as near VA, reading speed, central visual field, and several activities influencing quality of life. ${ }^{47}$ For the entire group of 30 eyes, the greatest improvement in quality of life was seen for reading ordinary print in newspapers. Reading speed increased significantly. It seemed that the improvement was greater for near activities than for distance activities.

Despite the therapeutic benefits of ranibizumab, repeated IVIs are required as often as every 4 weeks. A prospective clinical study evaluating the patients' perspective of treatment with ranibizumab, specifically pain, anxiety, and discomfort related to the ranibizumab IVI procedure, showed that IVI was less painful than anticipated $(P<0.001)$; 93\% were neither anxious nor fearful after the first IVI. ${ }^{48}$ Reducing anxiety and concerns about anticipated IVI-related discomfort or pain is likely to improve the patient experience and compliance with repeated procedures.

\section{Conclusion}

In conclusion, ranibizumab IVI has emerged as an effective treatment for wet AMD, which can greatly improve vision outcomes and quality of life. Clinical evidence indicates that ranibizumab initiation with three consecutive monthly injections is optimal, providing the greatest VA gain. After the initiation phase, the strongest evidence is for continued monthly treatment. If this is not feasible, a flexible individualized approach may achieve similar outcomes to monthly therapy, but it still needs to be verified. The flexible approach requires approximate monthly monitoring to capture signs of active disease and reinitiate treatment without delay. Overall, ranibizumab has been well tolerated in clinical trials, with a low incidence of ocular and systemic serious adverse events. However, clinical studies generally do not provide answers about the long-term safety of repeated IVI beyond 2 years of treatment. Moreover, because these clinical studies were conducted in a limited number of selected patients, they did not have sufficient power to detect relatively infrequent adverse events. As a widely licensed agent, the therapeutic value of ranibizumab can be assessed by use in everyday clinical practice. Ongoing and future studies with ranibizumab will provide further data on the best dosing regimens for optimal risk:benefit ratios. It is meaningful to determine whether less frequent injections on an as-needed schedule, based on monthly monitoring for signs of active disease, may have the most optimal risk:benefit ratio.

\section{Disclosure}

The authors report no conflicts of interest in this work.

\section{References}

1. Seddon JM, Chen CA. The epidemiology of age-related macular degeneration. Int Ophthalmol Clin. 2004;44:17-39.

2. Bressler NM. Age-related macular degeneration is the leading cause of blindness. JAMA. 2004;291:1900-1901.

3. Ferris FL 3rd, Fine SL, Hyman L. Age-related macular degeneration and blindness due to neovascular maculopathy. Arch Ophthalmol. 1984;102:1640-1642.

4. Funk M, Karl D, Georgopoulos M. Neovascular age-related macular degeneration: intraocular cytokines and growth factors and the influence of therapy with ranibizumab. Ophthalmology. 2009;116: 2393-2399.

5. Das A, McGuire PG. Retinal and choroidal angiogenesis: pathophysiology and strategies for inhibition. Prog Retin Eye Res. 2003;22:721-748.

6. Tong JP, Chan WM, Liu DT, et al. Aqueous humor levels of vascular endothelial growth factor and pigment epithelium-derived factor in polypoidal choroidal vasculopathy and choroidal neovascularization. Am J Ophthalmol. 2006;141:456-462.

7. Gaudreault J, Fei D, Rusit J, et al. Preclinical pharmacokinetics of ranibizumab (rhuFabV2) after a single intravitreal administration. Invest Ophthalmol Vis Sci. 2005;46:726-733.

8. Blick SKA, Keating GM, Wagstaff AJ. Ranibizumab. Drugs. 2007;67:1199-1206.

9. Lucentis (ranibizumab injection). Full prescribing information. South San Francisco, CA: Genentech, Inc; Jun 2010.

10. Ferrara N, Damico L, Shams N, et al. Development of ranibizumab, an anti-vascular endothelial growth factor antigen binding fragment, as therapy for neovascular age-related macular degeneration. Retina. 2006;26:859-870.

11. Ferrara N. Vascular endothelial growth factor and age-related macular degeneration: from basic science to therapy. Nat Med. 2010;16:1107-1111.

12. Lowe J, Araujo J, Yang J, et al. Ranibizumab inhibits multiple forms of biologically active vascular endothelial growth factor in vitro and in vivo. Exp Eye Res. 2007;85:425-430. 
13. European Medicines Agency. Assessment report for Lucentis (ranibizumab). Procedure no: EMEA/H/C/000715/II/0020 [online]. Available from URL: http://www.ema.europa.eu/docs/ en_GB/document_library/EPAR_-_Assessment_Report_-_Variation/ human/000715/WC500101009.pdf. Accessed May 17, 2012.

14. Tolentino M. Systemic and ocular safety of intravitreal antiVEGF therapies for ocular neovascular disease. Surv Ophthalmol. 2011;56(2):95-113.

15. Center for Drug Evaluation and Research. BLA application number: 125156. Clinical pharmacology and biopharmaceutics review(s) [online]. Available from URL: http://www.accessdata.fda.gov/ drugsatfda_docs/nda/2006/125156s0000_Lucentis_ClinPharmR.pdf. Accessed September 16, 2011.

16. Klein ML, Jorizzo PA, Watzke RC. Growth features of choroidal neovascular membranes in age-related macular degeneration. Ophthalmology. 1989;96:1416-1419.

17. Arias L, Armada F, Donate J, et al. Delay in treating age-related macular degeneration in Spain is associated with progressive vision loss. Eye. 2009;23:326-333.

18. Muether PS, Hermann MM, Koch K, et al. Delay between medical indication to anti-VEGF treatment in age-related macular degeneration can result in a loss of visual acuity. Graefe's Arch Clin Exp Ophthalmol. 2011;249:633-637.

19. Matthe E, Sandner D. Early treatment of exudative age-related macular degeneration with ranibizumab (Lucentis): the key to success. Ophthalmology. 2011;108:237-243.

20. Rosenfeld PJ, Brown DM, Heier JS, et al. Ranibizumab for neovascular age-related macular degeneration. $N$ Engl J Med. 2006;355: 1419-1431.

21. Kaiser PK, Brown DM, Zhang K, et al. Ranibizumab for predominantly classic neovascular age-related macular degeneration: subgroup analysis of first-year ANCHOR results. Am J Ophthalmol. 2007;144: $850-857$.

22. Brown DM, Michels M, Kaiser PK, et al. Ranibizumab versus verteporfin photodynamic therapy for neovascular age-related macular degeneration: two-year results of the ANCHOR study. Ophthalmology. 2009;116:57-65.

23. Regillo CD, Brown DM, Abraham P, et al. Randomized, doublemasked, sham-controlled trial of ranibizumab for neovascular agerelated macular degeneration: PIER study year 1. Am J Ophthalmol. 2008;145:239-248.

24. Abraham P, Yue H, Wilson L, et al. Randomized, double-masked, sham- controlled trial of ranibizumab for neovascular age-related macular degeneration: PIER Study Year 2. Am J Ophthalmol. 2010;150: 315-324.

25. Boyer DS, Heier JS, Brown DM, et al. A phase IIIb study to evaluate the safety of ranibizumab in subjects with neovascular agerelated macular degeneration. Ophthalmology. 2009;116: 1731-1739.

26. Schmidt-Erfurth U, Eldem B, Guymet R, et al. Efficacy and safety of monthly versus quarterly ranibizumab treatment in neovascular agerelated macular degeneration: the EXCITE study. Ophthalmology. 2011;118:831-839.

27. Fung AE, Lalwani GA, Rosenfeld PJ, et al. An optical coherence tomography-guided, variable dosing regimen with intravitreal ranibizumab (Lucentis) for neovascular age-related macular degeneration. Am J Ophthalmol. 2007;143:566-583.

28. Safety and efficacy of a flexible dosing regimen of ranibizumab in neovascular age-related macular degeneration: the SUSTAIN study. Ophthalmology. 2011;118:663-671.

29. CATT Research Group. Ranibizumab and bevacizumab for treatment of neovascular age-related macular degeneration: 2-year results. Ophthalmology. May 1, 2012. [Epub ahead of print.]
30. Monés J. A review of ranibizumab clinical trial data in exudative agerelated macular degeneration and how to translate it into daily practice. Ophthalmologica. 2011;225:112-119.

31. Kaiser PK, Brown DM, Zhang K, et al. Ranibizumab for predominantly classic neovascular age-related macular degeneration: subgroup analysis of first-year ANCHOR results. Am J Ophthalmol. 2007;144: $850-857$.

32. Amoaku W, National Institute for Health and Clinical Excellence. Ranibizumab: the clinician's guide to commencing, continuing, and discontinuing treatment. Eye (Lond). 2009;23:2140-2142.

33. Antoszyk AN, Tuomi L, Chung CY, et al. Ranibizumab combined with verteporfin photodynamic therapy in neovascular age-related macular degeneration. Am J Ophthalmol. 2008;145:862-874.

34. Schmidt-Erfurth U, Wolf S. Same-day administration of verteporfin and ranibizumab $0.5 \mathrm{mg}$ in patients with choroidal neovascularization due to age-related macular degeneration. Br J Ophthalmol. 2008;92: $1628-1635$.

35. Ley AM, et al. Combination therapy with verteporfin PDT and ranibizumab: twelve-month efficacy and safety results of the MONT BLANC study. Presented at AAO 2009; Abstract PA005.

36. Fintak DR, Shah GK, Blinder KJ, et al. Incidence of endophthalmitis related to intravitreal injection of bevacizumab and ranibizumab. Retina. 2008;28:1395-1399.

37. Koh A, Lim TH, Au Eong K, et al. Optimising the management of choroidal neovascularization in Asian patients: consensus on treatment recommendations for anti-VEGF therapy. Singapore Med J. 2011;52: $232-240$.

38. Chang LK, Sarraf D. Tears of the retinal pigment epithelium: an old problem in a new era. Retina. 2007;27:523-534.

39. Chiang A, Chang LK, Yu F, et al. Predictors of anti-VEGF-associated retinal pigment epithelial tear using FA and OCT analysis. Retina. 2008;28:1265-1269.

40. Ferrara N, Davis-Smyth T. The biology of vascular endothelial growth factor. Endocr Rev. 1997;18:4-25.

41. Gillies MC, Wong TY. Ranibizumab for neovascular age-related macular degeneration. $N$ Engl J Med. 2007;356:748-749; author reply 749-750.

42. Boyer DS, Chung CY, Tuomi L. A safety overview of ranibizumab in patients with wet AMD: ANCHOR, MARINA, PIER, and SAILOR Studies. Presented at the American Academy of Ophthalmology (AAO) 2008 Annual Meeting, Atlanta, Georgia, US. November 8-11, 2008.

43. Ueta T, Yanagi Y, Tamaki Y, et al. Cerebrovascular accidents in ranibizumab. Ophthalmology. 2009;116:362.

44. Fraser-Bell S, Wu J, Klein R, et al. Cardiovascular risk factors and age-related macular degeneration: the Los Angeles Latino Eye Study. Am J Ophthalmol. 2008;145:308-316.

45. Wilson PW, D'Agostino RB, Levy D, et al. Prediction of coronary heart disease using risk factor categories. Circulation. 1998;97: 1837-1847.

46. Singer MA, Awh CC, Sadda S, et al. HORIZON: an open-label extension trial of ranibizumab for choroidal neovascularization secondary to age-related macular degeneration. Ophthalmology. February 4, 2012. [Epub ahead of print.]

47. Frennesson C, Nilsson UL, Peebo BB, et al. Significant improvements in near vision, reading speed, central visual field and related quality of life after ranibizumab treatment of wet age-related macular degeneration. Acta Ophthalmol. 2010;88:420-425.

48. Chua PY, Mitrut I, Armbrecht AM, et al. Evaluating patient discomfort, anxiety, and fear before and after ranibizumab intravitreous injection for wet age-related macular degeneration. Arch Ophthalmol. 2009;127:939-940. 
Therapeutics and Clinical Risk Management

Dovepress

\section{Publish your work in this journal}

Therapeutics and Clinical Risk Management is an international, peerreviewed journal of clinical therapeutics and risk management, focusing on concise rapid reporting of clinical studies in all therapeutic areas, outcomes, safety, and programs for the effective, safe, and sustained use of medicines. This journal is indexed on PubMed Central, CAS,
EMBase, Scopus and the Elsevier Bibliographic databases. The manuscript management system is completely online and includes a very quick and fair peer-review system, which is all easy to use. Visit http://www.dovepress.com/testimonials.php to read real quotes from published authors.

Submit your manuscript here: http://www.dovepress.com/therapeutics-and-clinical-risk-management-journal 